\title{
MERCADO BRASILEIRO DE LEITE: CAUSALIDADE DE PREÇOS NOS PRINCIPAIS ESTADOS PRODUTORES
}

\author{
Angélica Pott de Medeiros, Bruna Márcia Machado Moraes, Reisoli Bender Filho
}

\begin{abstract}
RESUMO
O presente artigo tem como objetivo geral verificar o relacionamento entre os preços pagos aos produtores pelo leite in natura, nos estados de Minas Gerais, São Paulo, Goiás, Paraná, Bahia e Rio Grande do Sul, estados caracterizados como os principais produtores de leite no Brasil. Para tal, foi utilizado o teste de causalidade de Toda e Yamamoto (1995). Como principais resultados, foi identificado que os preços recebidos por Paraná e São Paulo recebem influência dos preços praticados nos outros estados. Além disso, Minas Gerais, que é o estado com maior representatividade na produção brasileira de leite, exerce influência nos preços pagos ao produtor de todos os outros estados brasileiros.

Palavras-chave: Preços de leite. Causalidade. Produção de leite.

\section{BRAZILIAN'S MILK MARKET: CAUSALITY OF PRICES AMONG KEY PRODUCERS STATES}

\begin{abstract}
This article has as its general objective to verify the relations between the prices paid to producers for fresh milk in the states of Minas Gerais, São Paulo, Goiás, Paraná, Bahia and Rio Grande do Sul states characterized as the main milk producers in Brazil.For this, was used the causality test of Toda and Yamamoto (1995). As main results, it was identified that the prices received by Parana and Sao Paulo are influenced by the prices charged in other states.Moreover, Minas Gerais, which is the state with the largest representation in the Brazilian milk production, influences the prices paid to producers of all other states.
\end{abstract}

Keywords: Milk prices. Causality. Milk production.

\section{INTRODUÇÃO}

A partir da década de 1950, a produção de leite deixou de ser realizada, em sua maioria, para a subsistência, passando a ser utilizada como fonte de renda, ocorrendo de forma paralela com o processo de industrialização do Brasil. Porém, até os anos 1990, o comércio de leite cru foi regulamentado pelas agências do governo, e os preços praticados em todas as regiões do país eram similares (BORTOLETO; WILKINSON, 2000).

Atualmente, os preços recebidos pelos produtores nos estados são diversificados, variando de $\mathrm{R} \$ 0,81$ a $\mathrm{R} \$ 0,99$ por litro, esse dado é referente ao preço pago aos produtores em janeiro de 2016, para os estados de Mato Grosso do Sul e São Paulo, respectivamente (CEPEA, 2016). Essa precificação depende, geralmente, da oferta e demanda, considerando tanto a safra e quanto a entressafra (BANKÚTI, 2009). Ribeiro et al. (2011) complementa, o preço pago ao produtor 
aumenta com a alta demanda pelo produto, e na época chuvosa, a oferta de leite aumenta, e seu preço diminui.

Em relação à produção, o Brasil está entre os maiores produtores de leite do mundo, o que se deve à sua extensão territorial, que propícia à agricultura e a pecuária, sendo que a atividade leiteira está presente em quase $90 \%$ das propriedades rurais do país, sendo que, a maioria realiza a atividade com fins de comércio, não apenas para subsistência (EMBRAPA, 2015).

A produção brasileira se apresenta bastante heterogênea, tanto em termos de mercado (produção, preços e consumo), quanto em termos de produtividade (formas de produção). A região sul é a principal produtora, sendo responsável por cerca de 34,7\% da produção brasileira, entre os estados, destacam-se o Rio Grande do Sul, responsável por 38,4\% da produção da região, e o Paraná com 37,2\%. Em seguida, vem a região sudeste, com 34,6\% da produção do país, seus estados contribuem significativamente à relevância produtiva da região, destaca-se o estado de Minas Gerais, responsável por $77 \%$ da produção regional. As demais regiões possuem produção mais baixa, a norte representa 5,5\%, a nordeste $11 \%$ e a região centro-oeste $14 \%$ (IBGE, 2015).

Tendo em vista a heterogeneidade do mercado do leite, o presente estudo busca verificar o relacionamento entre os preços pagos aos produtores pelo leite in natura, nos estados de Minas Gerais, São Paulo, Goiás, Paraná, Bahia e Rio Grande do Sul, estados caracterizados como os principais produtores de leite no Brasil, no período de período de janeiro de 2000 a dezembro de 2015.

Esse estudo avança na discussão ao propor a utilização de variáveis como o preço do leite dos principais estados produtores. De forma a complementar as análises encontradas na literatura, que discutem o comportamento de causalidade entre importações de leite em pó e UHT, preços no atacado e no varejo, preços aos produtores nos estados de Paraná, São Paulo, Minas Gerais, Rio Grande do Sul e Goiás, porém, até o ano de 2006, além de preços de rações para o gado leiteiro (BARROS et al., 2004; BARROS, DE LIMA, FERNANDES, 2010; DIAS et al., 2008; FERNANDES, BRAGA, DE LIMA, 2008; YAMAGUCHI, ARAÚJO, 2005).

Além desta introdução, o artigo está dividido em quatro seções, em seguida apresenta-se a revisão da literatura, na seção seguinte descreve-se a metodologia empregada no desenvolvimento do estudo. Posteriormente, nas últimas duas seções, são apresentados e analisados os resultados, e por fim delineadas as considerações finais. 


\section{PREÇOS DO LEITE NOS ESTADOS BRASILEIROS}

Ao passo em que o Brasil começou a ganhar visibilidade externa, nos anos 2000, a produtividade do setor lácteo aumentou, apresentando-se como o segundo maior exportador do produto. Mas, ao mesmo tempo, com o aumento da classe $\mathrm{C}$, e em consequência, aumento de consumidores de leite e derivados, a produção interna tornou-se insuficiente para suprir a demanda doméstica (CAMPOS, PIACENTI, 2007).

A produção de leite é realizada de diferentes formas, pois, apresenta sistemas de alto nível tecnológico, alta qualidade genética do rebanho, além de suplementação alimentar. Porém, com a propagação da agricultura familiar, onde se desenvolve um sistema produtivo de forma menos qualificada, e com padrões genéticos menos sofisticados, onde sua produção é destinada ao mercado informal (BORGES et al., 2014).

Como observado, o Brasil possui mercados dinâmicos e demograficamente diferentes em relação à produção de leite. Nas regiões sul e sudeste, o produto possui uma demanda menor, tendo em vista a denso número de empresas que beneficiam o produto, produzindo uma variedade maior de derivados, como o leite em pó, sem lactose, iogurtes, entre outros (SOCIEDADE NACIONAL DE AGRICULTURA, 2015). Além de que, nessas regiões, a atividade é desenvolvida, em sua maioria, em propriedades de pequeno porte, grande parte caracterizada como de agricultura familiar, próximas entre si, facilitando a captação pelas empresas (TRICHES, 2011).

Conforme exposto na Tabela 1, a região sul apresentou a maior representatividade na produção brasileira de leite, do que as demais regiões, com 34,7\% do total de litros produzidos em 2014. A região também apresenta o maior índice de produtividade por animal ordenhado, cerca de 2.789 litros por ano. Entre os estados, destaca-se o Rio Grande do Sul, com 38,4\% da produção da região sul, além disso, o estado se destaca na produtividade, a qual ultrapassa 3.000 litros por ano, apresentando a maior produtividade do país, porém, possui pouco rebanho, cerca de 6,7\%. Ainda, conforme Tabela 2, dentre os estados analisados, o Rio Grande do Sul, apresenta um dos menores preços líquidos, pagos aos produtores, cerca de $\mathrm{R} \$ 0,887$ por litro, registrado em fevereiro de 2016. Em relação ao estado do Paraná, apesar de ter uma produtividade menor, cerca de 2.629 litros/ano, sua participação no rebanho é de 7,5\%, ou seja, o estado possui um rebanho 
maior, sua participação na produção regional é de $37,2 \%$, e o preço pago ao produtor é cerca de $\mathrm{R} \$ 0,936 /$ litro.

Tabela 1- Produtividade e participação das regiões e estados na produção de leite e no rebanho - 2014.

\begin{tabular}{|c|c|c|c|c|}
\hline Região/Estados & $\begin{array}{c}\text { Part. } \\
\text { Produção } \\
\text { Brasil }\end{array}$ & $\begin{array}{c}\text { Part. } \\
\text { Produção } \\
\text { Região }\end{array}$ & $\begin{array}{c}\text { Part. } \\
\text { Rebanho } \\
\text { Brasil } \\
\end{array}$ & $\begin{array}{c}\text { Produtividade } \\
\text { (l/ano) }\end{array}$ \\
\hline Norte & $5,5 \%$ & - & $9,6 \%$ & 876 \\
\hline Rondônia & $2,7 \%$ & $48,3 \%$ & $3,4 \%$ & 1217 \\
\hline Acre & $0,1 \%$ & $2,7 \%$ & $0,4 \%$ & 638 \\
\hline Amazonas & $0,1 \%$ & $2,6 \%$ & $0,5 \%$ & 465 \\
\hline Roraima & $0,0 \%$ & $0,6 \%$ & $0,1 \%$ & 345 \\
\hline Pará & $1,6 \%$ & $28,5 \%$ & $3,2 \%$ & 746 \\
\hline Amapá & $0,0 \%$ & $0,6 \%$ & $0,1 \%$ & 837 \\
\hline Tocantins & $0,9 \%$ & $16,7 \%$ & $2,0 \%$ & 695 \\
\hline Nordeste & $11,1 \%$ & - & $20,6 \%$ & 818 \\
\hline Maranhão & $1,1 \%$ & $10,1 \%$ & $2,7 \%$ & 631 \\
\hline Piauí & $0,2 \%$ & $2,1 \%$ & $0,6 \%$ & 571 \\
\hline Ceará & $1,4 \%$ & $12,7 \%$ & $2,5 \%$ & 851 \\
\hline Rio Grande do Norte & $0,7 \%$ & $6,0 \%$ & $1,1 \%$ & 904 \\
\hline Paraíba & $0,5 \%$ & $4,4 \%$ & $0,9 \%$ & 797 \\
\hline Pernambuco & $1,9 \%$ & $16,9 \%$ & $2,0 \%$ & 1396 \\
\hline Alagoas & $0,9 \%$ & $7,8 \%$ & $0,7 \%$ & 1887 \\
\hline Sergipe & $1,0 \%$ & $8,9 \%$ & $1,0 \%$ & 1466 \\
\hline Bahia & $3,4 \%$ & $31,2 \%$ & $9,0 \%$ & 586 \\
\hline Sudeste & $34,6 \%$ & - & $34,4 \%$ & 1533 \\
\hline Minas Gerais & $26,6 \%$ & $77,0 \%$ & $25,2 \%$ & 1613 \\
\hline Espírito Santo & $1,4 \%$ & $4,0 \%$ & $1,8 \%$ & 1158 \\
\hline Rio de Janeiro & $1,5 \%$ & $4,4 \%$ & $1,8 \%$ & 1281 \\
\hline São Paulo & $5,1 \%$ & $14,6 \%$ & $5,6 \%$ & 1380 \\
\hline Sul & $34,7 \%$ & - & $19,0 \%$ & 2789 \\
\hline Paraná & $12,9 \%$ & $37,2 \%$ & $7,5 \%$ & 2629 \\
\hline Santa Catarina & $8,5 \%$ & $24,5 \%$ & $4,8 \%$ & 2694 \\
\hline Rio Grande do Sul & $13,3 \%$ & $38,4 \%$ & $6,7 \%$ & 3034 \\
\hline Centro-Oeste & $14,1 \%$ & - & $16,4 \%$ & 1315 \\
\hline Mato Grosso do Sul & $1,5 \%$ & $10,6 \%$ & $2,2 \%$ & 1022 \\
\hline Mato Grosso & $2,1 \%$ & $14,5 \%$ & $2,5 \%$ & 1243 \\
\hline Goiás & $10,5 \%$ & $74,1 \%$ & $11,5 \%$ & 1386 \\
\hline
\end{tabular}


Fonte: IBGE (2015), adaptado pelos autores.

\begin{tabular}{l|l} 
Distrito Federal & $0,1 \%$ \\
\hline
\end{tabular}

$0,7 \%$

$0,1 \%$

1485

Em seguida, em termos de produção, temos a região sudeste, com 34,6\% da produção, entre seus estados, destaca-se o estado de Minas Gerais, responsável por cerca de $77 \%$ da produção da região, além disso, o estado possui o maior rebanho do país, 25,2\%, e uma produtividade de 1.613 litros/ano. Os estados de São Paulo e Minas Gerais apresentam o maior preço pago ao produtor dentro os estados pesquisados (Tabela 2), cerca de $\mathrm{R} \$ 1,018$ e $\mathrm{R} \$ 1,005$, respectivamente.

Já na região centro-oeste, a participação foi de aproximadamente $14 \%$, sendo o estado de Goiás o de maior representatividade na produção, 74,1\%, seguido por Mato Grosso, cerca de $15 \%$ da produção da região. Tais estados possuem 11,5\% e 2,5\% do rebanho brasileiro, respectivamente. A região possui uma produtividade de 1315 litros por ano, apesar da baixa produção, o Distrito Federal, apresenta a maior produtividade da região, 1.485 litro/ano. O estado de Goiás apresenta um preço pago ao produtor de R $\$$ 0,971, já Mato Grosso do Sul, dentre os pesquisados, possui o menor preço bruto e preço pago ao produtor, $\mathrm{R} \$ 0,943$ e $\mathrm{R} \$ 0,825$, respectivamente.

A estrutura produtiva da região nordeste é variada, a região representa cerca de $11 \%$ da produção do país, dentre os seus estados, a Bahia possui a maior produção, 31,2\% em relação a região, possuindo 9\% do rebanho, o estado, apresenta um preço pago ao produtor de $\mathrm{R} \$ 0,957$. Em relação à produtividade, a região possui a menor produtividade, 818 litros por ano, porém a região possui estados que apresentam produtividade de 571 litros/ano, caso do Piauí, até 1.887 litros/ano, caso de Alagoas.

Com uma participação menos expressiva, está a região norte, com 5,5\% da produção brasileira. Dentre seus estados, destacam-se o estado de Rondônia, com 48,3\% da produção regional, e o Pará com 28,5\%. Além da produção, a região também possui baixa representatividade no rebanho, cerca de 10\%. O norte possui o estado com a menor produtividade, caso de Roraima, com 345 litros/ano, a média da região é de 876 litros por ano, sendo que o estado de Rondônia possui uma produtividade de 1.217 litros/ano.

\section{Tabela 2 - Preço bruto e líquido do litro do leite por estado - Fevereiro 2016.}


Fonte: CEPEA (2016), adaptado pelos autores.

\begin{tabular}{l|c|c}
\hline Estado & $\begin{array}{c}\text { Preço } \\
\text { Bruto }\end{array}$ & $\begin{array}{c}\text { Preço } \\
\text { Líquido }\end{array}$ \\
\hline São Paulo & 1,093 & 1,018 \\
Minas Gerais & 1,093 & 1,005 \\
Goiás & 1,072 & 0,971 \\
Espírito Santo & 1,057 & 0,973 \\
Rio de Janeiro & 1,034 & 0,977 \\
Bahia & 1,032 & 0,957 \\
Paraná & 1,024 & 0,935 \\
Ceará & 1,020 & 0,938 \\
Santa Catarina & 1,014 & 0,936 \\
Rio Grande do Sul & 0,972 & 0,887 \\
Mato Grosso do Sul & 0,943 & 0,825 \\
\hline
\end{tabular}

Conforme exposto na Tabela 2, os estados possuem preços diferentes entre si, isso se deve ao fato que a precificação do leite depende da oferta e da demanda, determinando o preço de safra e entressafra, ou seja, o preço que é pago ao produtor aumenta com o crescimento da demanda, e diminui com o aumento da oferta (BANKÚTI, 2009; RIBEIRO et al., 2011). O sistema de formação de preços em uma economia é extremamente complexo, já que depende da oferta e da demanda de determinado bem, e estes são determinados por outras variáveis que influenciam positiva e negativamente seus volumes. Cada consumidor monta sua cesta de necessidades, esperando que suas utilidades sejam maximizadas com um menor custo possível (ARRAES, SOUZA FILHO, 2008).

\subsection{Evidências empíricas}

Os estudos relacionados ao preço do leite se concentram em verificar a sua relação às importações (BARROS et al., 2004), ao Markup da indústria e varejo (BARROS, DE LIMA, FERNANDES, 2010), entre os estados (DIAS et al., 2008; FERNANDES, BRAGA, DE LIMA, 2008), e preços de rações (YAMAGUCHI, ARAÚJO, 2005). Com o objetivo de apresentar um panorama geral acerca do preço do leite, elaborou-se um quadro-resumo com os principais estudos, focando na discussão e nos resultados (ver Quadro 1). 


\section{Quadro 1 - Estudos anteriores}

\begin{tabular}{|c|c|c|c|}
\hline Estudos & Método & Variáveis & Resultados \\
\hline $\begin{array}{l}\text { Barros et al. } \\
(2004)\end{array}$ & $\begin{array}{l}\text { Teste de } \\
\text { Causalidade de } \\
\text { Granger }\end{array}$ & $\begin{array}{l}\text { Importação leite em pó, } \\
\text { Importação leite em fluido } \\
\text { Relação de preço leite em pó (preço } \\
\text { atacado e varejo), Preço pago ao } \\
\text { produtor, } \\
\text { Relação de preço leite em fluido (preço } \\
\text { atacado e varejo). O período de análise } \\
\text { foi de agosto de } 1994 \text { a dezembro de } \\
2003 \text {. }\end{array}$ & $\begin{array}{l}\text { Há bicausalidade entre o volume importado e a relação de preços no caso } \\
\text { do leite fluido. No caso de leite em pó, não se detectou causalidade entre } \\
\text { volume e preços relativos. } \\
\text { Os preços do leite UHT e ao produtor apresentam uma relação bicausal, } \\
\text { assim como os preços ao atacado e varejo do leite UHT. O preço do leite } \\
\text { em pó não mostrou nenhuma relação de causalidade. }\end{array}$ \\
\hline $\begin{array}{l}\text { Barros, De } \\
\text { Lima, } \\
\text { Fernandes } \\
(2010)\end{array}$ & $\begin{array}{ll}\text { Teste } & \text { de } \\
\text { causalidade } & \text { de } \\
\text { Granger } & \end{array}$ & $\begin{array}{l}\text { Preços ao Produtor, Markup da Indústria } \\
\text { e Markup do Varejo, no período de } 1998 \\
\text { a } 2007 .\end{array}$ & $\begin{array}{l}\text { Os markups da indústria e do varejo são responsáveis pelos preços pagos } \\
\text { aos produtores. Porém, o markup do varejo e o preço pago ao produtor } \\
\text { também causam, no sentido de Granger, o markup da indústria. Por último, } \\
\text { tanto produtor quanto indústria causam o markup do varejo. }\end{array}$ \\
\hline $\begin{array}{l}\text { Dias et al. } \\
(2008)\end{array}$ & $\begin{array}{ll}\text { Teste } & \text { de } \\
\text { causalidade } & \text { de } \\
\text { Granger } & \end{array}$ & $\begin{array}{l}\text { Preços recebidos pelos produtores nos } \\
\text { estados de Paraná, São Paulo, Minas } \\
\text { Gerais, Rio Grande do Sul e Goiás, no } \\
\text { período janeiro de } 1995 \text { a julho de } 2006 \text {. }\end{array}$ & $\begin{array}{l}\text { Causalidade bidirecional entre São Paulo e Minas Gerais; Goiás causa São } \\
\text { Paulo e Paraná, e o Paraná causa o preço do Rio Grande do Sul. }\end{array}$ \\
\hline $\begin{array}{l}\text { Fernandes, } \\
\text { Braga, De } \\
\text { Lima (2008) }\end{array}$ & $\begin{array}{l}\text { Modelo Vetorial } \\
\text { de Correção de } \\
\text { Erro (VEC). }\end{array}$ & $\begin{array}{l}\text { Séries mensais de preços de leite aos } \\
\text { produtores dos estados de Minas Gerais, } \\
\text { Goiás, Paraná, Rio Grande do Sul e São } \\
\text { Paulo, no período de } 1997 \text { a } 2005 \text {. }\end{array}$ & $\begin{array}{l}\text { Os resultados evidenciaram que a variação de } 1 \% \text { nos preços recebidos } \\
\text { pelos produtores de leite de Minas Gerais leva a uma variação de } 0,38 \% \text {, } \\
0,72 \%, 0,93 \% \text { e } 0,26 \% \text { nos preços em São Paulo, Rio Grande do Sul, } \\
\text { Paraná e Goiás, respectivamente. }\end{array}$ \\
\hline $\begin{array}{l}\text { Yamaguchi, } \\
\text { Araújo (2005) }\end{array}$ & $\begin{array}{ll}\text { Teste } & \text { de } \\
\text { causalidade } & \text { de } \\
\text { Sims, } & \end{array}$ & $\begin{array}{l}\text { Séries mensais de preços de leite } \\
\text { recebidos pelos produtores de leite tipos } \\
\text { "B" e "C" no Estado de São Paulo, e } \\
\text { preços de rações para vacas leiteiras } \\
\text { pagos pelos produtores, no período de } \\
\text { janeiro de } 1990 \text { a novembro de } 2004 \text {. }\end{array}$ & $\begin{array}{l}\text { Relação causal entre as variáveis "preço de leite" e "preço de ração" e } \\
\text { também "preço de ração" e "preço de leite", indicando relação causal } \\
\text { bidirecional, para ambos os tipos de leite, "B" e "C". }\end{array}$ \\
\hline
\end{tabular}

Fonte: Elaborado pelos autores.

\begin{tabular}{lr}
\hline Volume 5, Número 10 & Revista UNEMAT de Contabilidade \\
Jul./Dez. 2016 & 143
\end{tabular}


Diante do exposto, verifica-se que de acordo com Barros et al. (2004), há causalidade bidirecional entre o volume importado de leite em fluído e o seu preço, ainda, verificou-se também causalidade bidirecional entre o preço do leite e ao preço pago ao produtor. Já Barros, de Lima e Fernandes (2010), verificaram que causalidade bidirecional entre o markup da indústria, do varejo, e o preço pago aos produtores. Levando em consideração os insumos, Yamaguchi e Araújo (2005), constataram causalidade bidirecional entre o preço do leite e preço da ração.

De forma mais regional, Dias et al. (2008) constatou que há causalidade bidirecional entre São Paulo e Minas Gerais, e que Goiás causa São Paulo e Paraná, e o Paraná causa o preço do Rio Grande do Sul. Ao inverso de Fernandes, Braga e de Lima (2008), que verificaram que a variação em $1 \%$ nos preços pagos aos produtores de Minas Gerais, acarreta a uma variação de $0,38 \%, 0,72 \%, 0,93 \%$ e $0,26 \%$, aos estados de São Paulo, Rio Grande do Sul, Paraná e Goiás, respectivamente.

\section{ASPECTOS METODOLÓGICOS}

\subsection{Modelo Teórico}

Estudos que envolvem o conceito de causalidade têm se mostrado de extrema relevância para o entendimento de relações entre fatores econômicos, estando presentes em uma extensa bibliografia. Entretanto, diferentes definições de causalidade tem sido desenvolvidas (MURAKAMI, 2011).

A definição geral de causalidade foi estabelecida por Granger (1969), o qual diz que, para cada $\Omega_{t}$ representando toda a informação disponível de determinada variável de uma economia até o período t, $Y_{t}$ causa $X_{t+1}$ se, para algum $A$ :

$$
\operatorname{Pr}\left(X_{t+1} \in A \mid \Omega_{t}\right) \neq \operatorname{Pr}\left(X_{t+1} \in A \mid \Omega_{t}-Y_{t}\right)
$$

Nesse sentido, há causalidade de $Y_{t}$ sobre $X_{t+1}$ se a probabilidade é alternada quando são consideradas todas as informações disponíveis ou todo o conjunto sendo retirado a informação $Y_{t}$. Desta forma, a causalidade de Granger baseia-se na noção de precedência temporal: se uma variável $Y$ causa uma variável $X$, a variável $Y$ deve preceder a variável $X$. Neste caso, a variável $X$ deve ajudar a prever o valor desta 
variável $Y$. Estatisticamente, de acordo com Engle e Granger (1987), para a realização do teste, todas as variáveis devem ser estacionárias, ou os coeficientes de interesse multiplicam as variáveis estacionárias.

A estatística Wald individual tem uma distribuição assintótica se $\varepsilon$ é não singular. Se as variáveis de processo VAR são estacionárias, os estimadores OLS e a estatística Wald são válidas. No entanto, se as variáveis contêm raízes unitárias, então a estatística de Wald com base em estimativa de MQO no modelo VAR em nível tem distribuições assintóticas não padrão que podem envolver parâmetros não significativos. Portanto, o teste de causalidade de Granger não é válido para as variáveis não estacionárias (EMIRMAHMUTOGLU, KOSE, 2011).

Formalmente, o teste de causalidade de Granger (1969), tem como base as seguintes equações:

$$
\begin{aligned}
& P_{i t}=\sum \alpha_{i} P_{i t-k}^{*}+\sum \beta_{j} P_{i t-j}+\mu_{1 t} \\
& P_{i t}^{*}=\sum \lambda_{i} P_{i t-k}^{*}+\sum \delta_{j} P_{i t-j}+\mu_{2 t}
\end{aligned}
$$

em que $P_{i t}$ e $P_{i t}^{*}$ preços domésticos e internacional do produto $i$ no tempo $\mathrm{t} ; P_{i t-j}$ preço domésticos defasados em $j$ períodos; e ${ }^{P_{i t-k}^{*}}$ preços internacionais defasados em $k$ períodos. E ainda, supõe-se que $\mu_{1 t}$ e $\mu_{2 t}$ não sejam correlacionados e que o teste de causalidade seja realizado em séries estacionárias.

Assim, segundo Stock e Watson (2004), os possíveis resultados são:

a) Causalidade unidirecional de $P_{i t}^{*}$ para $P_{i t}$ : será indicada se os coeficientes estimados das defasagens de $P$ foram estatisticamente diferentes de zero como grupo e o conjunto de coeficientes estimados não for estatisticamente diferente de zero;

b) Causalidade unidirecional de $P_{\text {it para }} P_{i t}^{*}$ : se o conjunto de coeficientes defasados não é estatisticamente diferente de zero e o conjunto dos coeficientes é estatisticamente diferente de zero;

c) Causalidade bilateral: será sugerido quando os conjuntos dos coeficientes forem estatisticamente diferentes de zero em ambas as regressões;

d) Independência: ocorrerá quando os conjuntos de coeficientes não forem estatisticamente significativos em nenhuma das regressões. 
Conforme discutido, o teste de Granger exige o atendimento de tais premissas, as quais muitas vezes limitam a utilização do teste. Para minimizar essas limitações, Toda e Yamamoto (1995) propuseram uma abordagem alternativa simples para restrições de coeficiente de testes de um modelo VAR em nível. Em outras palavras, esse teste realiza procedimento que requer a estimação de um VAR aumentado, o que garante a distribuição assintótica da estatística de Wald ( $x^{2}$ distribuição assintótica), uma vez que o procedimento de teste é robusto para as propriedades de integração e cointegração do processo (ALIMI, OFONYELU, 2013).

Assim, o referido teste pode ser aplicado a uma série não estacionária para determinar a direção da causalidade. Toda e Yamamoto (1995) afirmam que, se a ordem máxima da integração da série (d-max) é adicionada ao modelo de Granger (1969), ele pode ser aplicado para o nível de série não estacionária e fornecer estimativas válidas. $\mathrm{O}$ modelo para testar a causalidade consiste nas seguintes formulações:

$$
\begin{aligned}
& X_{t}=c_{1}+\sum_{j=1}^{k+d} \alpha_{1 j} X_{t-j}+\sum_{j=1}^{k+d} \beta_{1 j} Y_{t-j}+\varepsilon_{1 t} \\
& Y_{t}=c_{2}+\sum_{j=1}^{k+d} \alpha_{2 j} Y_{t-j}+\sum_{j=1}^{k+d} \beta_{2 j} X_{t-j}+\varepsilon_{2 t}
\end{aligned}
$$

em que $c_{1}$ e $C_{\mathbf{z}}$ são as constantes, $k$ é a melhor ordem do vetor autorregressivo (VAR) do modelo, $Y$ e $X$ são séries não estacionárias, $\varepsilon_{1 t}$ e $\varepsilon_{\mathbf{z}}$ são ruído branco. Desse modo, $Y$ causa $X$ se $\beta_{1 j}$ não forem conjuntamente iguais à zero. Da mesma forma que $X$ causa $Y$ se $\beta_{z j}$ não forem conjuntamente iguais a zero. Os testes sobre os coeficientes são obtidos por um teste de Wald padrão (SULKU, 2011).

Segundo Fochezatto, Koshiyama e Alencastro (2010), para a aplicação do teste de Toda e Yamamoto, é necessária a realização de três etapas fundamentais. Primeiramente, deve-se definir o número ótimo de defasagens $(z)$ e a ordem máxima de integração do sistema (e). Posteriormente a isso, é estimado um VAR em níveis com um total de $(z+e)$ defasagens, conforme mostram as equações (6) e (7):

$$
\begin{aligned}
& Y_{t}=\alpha_{1}+\sum_{i=1}^{z} \beta_{1 i} Y_{t-1}+\sum_{j=z+1}^{z+e} \beta_{1 j} Y_{t-j}+\sum_{i=1}^{z} \gamma_{1 i} X_{t-1}+\sum_{j=z+1}^{z+e} \gamma_{1 j} X_{t-j}+\varepsilon_{1 t} \\
& X_{t}=\alpha_{2}+\sum_{i=1}^{z} \beta_{2 i} Y_{t-i}+\sum_{j=z+1}^{z+e} \beta_{2 j} Y_{t-j}+\sum_{i=1}^{z} \gamma_{2 i} X_{t-i}+\sum_{j=z+1}^{z+e} \gamma_{2 j} X_{t-j}+\varepsilon_{2 t}(7)
\end{aligned}
$$


O terceiro passo é a aplicação de um teste de restrição de Wald nos z primeiros coeficientes para testar a hipótese de não causalidade. O resultado mostrará se que há causalidade unidirecional de $X$ para $Y$ se a hipótese $H_{0}: \gamma_{1 i}=0$ for rejeitada e a hipótese $H_{0}: \beta_{2 i}=0$ não for rejeitada. Da mesma forma que haverá causalidade unidirecional de $Y$ para $X$ se a hipótese $H_{0}: \beta_{2 i}=0$ for rejeitada e a hipótese $H_{0}: \gamma_{1 i}=0$ não for.

\subsection{Modelo empírico}

Após especificado o modelo teórico, as equações a seguir indicam as possíveis relações de precedências entre os preços de leite in natura pago ao produtor dos estados mais intensivos em produção leiteira no Brasil. Para tanto, primeiramente são apresentadas as equações do modelo bivariado, em que são estimadas as relações de causalidade aos pares.

Nesse caso, como apresentado em(8), a série de dados de preços pago ao produtor do estado da Bahia foi analisada com relação à série de preços praticados no estado de Goiás. Assim, foi possível identificar se há precedência de causalidade no comportamento de preços entre os dois estados. Posterior a isso, foram analisados os preços da Bahia com relação aos preços de todos os outros estados. O mesmo foi realizado com os demais estados, conforme apresentado nas equações 9, 10, 11, 12 e 13.

$$
\begin{aligned}
& B a_{t}=\alpha_{1}+\sum_{i=1}^{z} \beta_{1 i} B a_{t-1}+\sum_{j=z+1}^{z+e} \beta_{1 j} B a_{t-j}+\sum_{i=1}^{z} \gamma_{1 i} G o_{t-1}+\sum_{j=z+1}^{z+e} \gamma_{1 j} G o_{t-j}+\varepsilon_{1 t} \\
& G o_{t}=\alpha_{2}+\sum_{i=1}^{z} \gamma_{2 i} G o_{t-1}+\sum_{j=z+1}^{z+e} \gamma_{2 j} G o_{t-j}+\sum_{i=1}^{z} \beta_{2 i} B a_{t-1}+\sum_{j=z+1}^{z+e} \beta_{2 j} B a_{t-j}+\varepsilon_{2 t} \\
& M g_{t}=\alpha_{3}+\sum_{i=1}^{z} \varphi_{3 i} M g_{t-1}+\sum_{j=z+1}^{z+e} \varphi_{3 j} M g_{t-j}+\sum_{i=1}^{z} \gamma_{3 i} G o_{t-1}+\sum_{j=z+1}^{z+e} \gamma_{3 j} G o_{t-j}+\varepsilon_{3 t} \\
& R s_{t}=\alpha_{4}+\sum_{i=1}^{z} \theta_{4 i} R s_{t-1}+\sum_{j=z+1}^{z+e} \theta_{4 j} R s_{t-j} \sum_{i=1}^{z} \phi_{4 i} \operatorname{Pr}_{t-1}+\sum_{j=z+1}^{z+e} \phi_{4 j} \operatorname{Pr}_{t-j}+\varepsilon_{4 t} \\
& \operatorname{Pr}_{t}=\alpha_{5}+\sum_{i=1}^{z} \phi_{5 i} \operatorname{Pr}_{t-1}+\sum_{j=z+1}^{z+e} \phi_{5 j} \operatorname{Pr}_{t-j}+\sum_{i=1}^{z} \varphi_{5 i} M g_{t-1}+\sum_{j=z+1}^{z+e} \varphi_{5 j} M g_{t-j}+\varepsilon_{5 t} \\
& S p_{t}=\alpha_{6}+\sum_{i=1}^{z} \varpi_{6 i} S p_{t-1}+\sum_{j=z+1}^{z+e} \varpi_{6 j} S p_{t-j}+\sum_{i=1}^{z} \theta_{6 i} R s_{t-1}+\sum_{j=z+1}^{z+e} \theta_{6 j} R s_{t-j}+\varepsilon_{6 t}
\end{aligned}
$$


Após estimadas as relações aos pares entre os estados analisados (Bahia, Goiás, São Paulo, Paraná, Rio Grande do Sul e Minas Gerais), foram analisadas as séries de preços em conjunto. Assim, dando origem ao modelo multivariado, onde cada série de preços foi analisada com relação às séries de preços dos demais estados.

\subsection{Procedimentos econométricos e dados}

Para testar a presença ou não de raiz unitária na série e ordem de integração, foi utilizado o Teste de Dickey-Fuller Aumentado - ADF, desenvolvido por Dickey e Fuller (1981), que tem como base a seguinte expressão:

$$
\Delta Y_{t}=\beta+\delta T+\gamma Y_{t-1}+\alpha_{i} \sum_{i=1}^{m} \Delta Y_{t-1}+\varepsilon_{t}
$$

em que $\beta$ é o intercepto; $\delta$ té a tendência; $\Delta$ é o operador diferença.

Por meio do teste de Dickey-Fuller Aumentado, é possível identificar também a ordem máxima de integração do sistema, $e_{\max }$, a partir das propriedades univariadas das séries temporais (FOCHEZATTO, KOSHIYAMA, ALENCASTRO, 2009). Após realizado o teste de raiz unitária e definida a ordem de integração, foi estimado o VAR e testado o procedimento de Toda e Yamamoto.

Os dados foram obtidos por meio da base do Centro de Pesquisas em Economia Aplicada - CEPEA/Leite. Eles correspondem aos preços brutos, considerando frete e impostos, sendo aquele recebido pelo produtor por litro comercializado de leite in natura, e possuem periodicidade mensal.

Além disso, os preços são calculados em cada mesorregião dos estados participantes da pesquisa realizada pelo CEPEA/Leite para a determinação dos preços, que são ${ }^{1}$ Rio Grande do Sul, Paraná, São Paulo, Minas Gerais, Goiás e Bahia. A partir dos dados coletados, foi realizada a ponderação nos preços médios de acordo com a participação da produção formal de cada estado no total da produção de todos os estados.

\footnotetext{
${ }^{1}$ Santa Catarina também faz parte dos estados utilizados como base de análise, porém, só a partir de julho de 2005. Assim, não foi incluído na análise.
} 
Para a estimação do modelo de causalidade, os dados foram deflacionados a partir da tabela de atualização de valores divulgados pela FEE (2016). Da mesma forma que identificado comportamento sazonal na série, sendo necessário o ajuste através do método Census X12².

\section{ANÁLISE DOS RESULTADOS}

Com o objetivo de analisar a relação de preços do leite in natura nos principais estados produtores, na Figura 1 estão expostos os preços observados nas regiões brasileiras, no período de janeiro de 2000 a dezembro de 2015. Os estados participantes referem-se aqueles pesquisados pelo CEPEA/Leite à análise mensal de preços, quais sejam: Goiás, Minas Gerais, Rio Grande do Sul, São Paulo, Paraná e Bahia.

\section{Figura 1: Evolução dos preços pagos aos produtores de leite in natura nos principais estados produtores.}

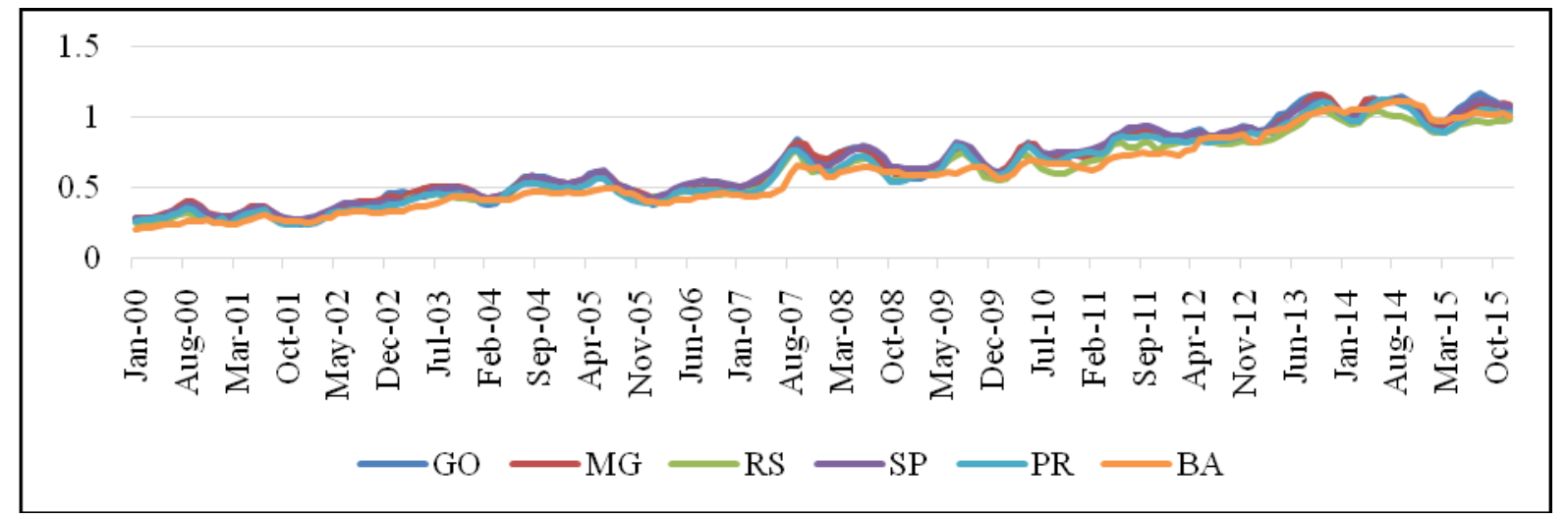

Fonte: Elaborado pelos autores a partir de Cepea/Leite (2016).

Como observado, os preços dos principais estados produtores de leite in natura, apresentaram comportamento semelhante ao longo do período analisado. Exceção foram os estados do Rio Grande do Sul e Bahia que, em alguns momentos, apresentaram trajetória distinta daquela observada para os outros estados.

Para analisar estatisticamente as séries de preços do leite dos estados selecionados, foi realizado o teste de estacionariedade ADF. Os resultados demonstram

\footnotetext{
${ }^{2}$ Detalhes ver U.S. Bureau oftheCensus (2015).
} 
que as séries apresentaram tendência estocástica, em que, segundo Greene (2002), terão a característica de retornar para sua média com o passar do tempo. Nesse caso, a série tenderá a apresentar um comportamento não estacionário. Para tal, foram analisadas as séries com constante e tendência, como exposto na Tabela 3.

Tabela 3: Resultados do Teste ADF para os estados selecionados, no período de 2000 a 2015.

\begin{tabular}{c|c|c|c|c}
\cline { 2 - 4 } & \multicolumn{3}{c}{ Nível Primeira Diferença } \\
\hline Variável & $\tau_{t}$ & $\tau_{\mu}$ & $\tau_{t}$ & $\tau_{\mu}$ \\
\hline p_ba & -1.718555 & 2.859504 & $-12.32286 * * *$ & - \\
\hline p_go & $-3.663941^{* * *}$ & - & - & - \\
\hline p_mg & $-4.076123 * * *$ & - & - & - \\
\hline p_pr & $-3.192850^{* * *}$ & $-4.592456 * * *$ & - & - \\
\hline p_pr & $-3.883563 * * *$ & - & - & - \\
\hline p_sp & $-3.363271 * *$ & $-4.703514 * * *$ & & - \\
\hline
\end{tabular}

Fonte: Elaborado pelos autores (2016).

*, **, ***Indica que a hipótese nula é rejeitada a um nível de significância de 10\%, 5\% e $1 \%$ respectivamente.

Pode-se identificar que a série de preços do leite recebido pelo produtor no estado da Bahia foi estacionária em primeira diferença a um nível de $5 \%$ de significância. Diferentemente das outras séries que foram estacionárias em nível e com constante, como é o caso das séries de preços de Goiás, Minas Gerais e Rio Grande do Sul. Já os preços dos estados do Paraná e de São Paulo foram estacionários em nível, porém com a presença de tendência.

A partir do teste de estacionariedade, identificou-se 1 como o nível máximo de integração da série, limite utilizado para estabelecer o VAR antes dos procedimentos do teste de causalidade de Toda e Yamamoto (1995). Assim sendo, foi estabelecido $e=1$.O

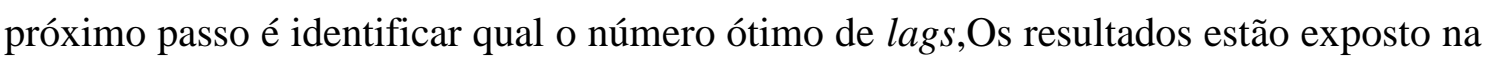
Tabela 4. 
Tabela 4: Resultados dos testes do comprimento de lags.

\begin{tabular}{ccccccc}
\hline Lag & LogL & LR & FPE & AIC & SC & HQ \\
\hline 0 & 1802.794 & NA & $1.33 \mathrm{e}-16$ & -19.53037 & -19.42553 & -19.48788 \\
1 & 2575.107 & 1485.862 & $4.44 \mathrm{e}-20$ & -27.53377 & $\mathbf{- 2 6 . 7 9 9 9 2 *}$ & -27.23633 \\
2 & 2660.221 & 158.2012 & $2.61 \mathrm{e}-20$ & -28.06762 & -26.70476 & $-27.51524^{*}$ \\
3 & 2700.671 & 72.54581 & $2.49 \mathrm{e}-20^{*}$ & $-28.11598^{*}$ & -26.12412 & -27.30866 \\
4 & 2731.744 & $53.70257^{*}$ & $2.64 \mathrm{e}-20$ & -28.06243 & -25.44156 & -27.00016 \\
5 & 2758.617 & 44.69187 & $2.95 \mathrm{e}-20$ & -27.96323 & -24.71335 & -26.64601 \\
6 & 2788.662 & 48.00561 & $3.19 \mathrm{e}-20$ & -27.89850 & -24.01961 & -26.32633 \\
7 & 2817.469 & 44.14999 & $3.51 \mathrm{e}-20$ & -27.82031 & -23.31241 & -25.99320 \\
8 & 2844.471 & 39.62247 & $3.98 \mathrm{e}-20$ & -27.72251 & -22.58560 & -25.64045 \\
\hline
\end{tabular}

Fonte: Elaborado pelos autores (2016).

LR: sequential modified LR test statistic (each test at 5\% level)

FPE: Final prediction error

AIC: Akaike information criterion

SC: Schwarz information criterion

HQ: Hannan-Quinn information criterion

Fonte: Resultado do trabalho. Elaborado pelos autores.

O teste indica a presença de um lag, conforme critério de Schwarz, que, como sugerido por Stock e Watson (2004), trata-se de um modelo mais robusto e parcimonioso, dado que os demais indicaram duas e três defasagens, o que resultaria em grande perda de graus de liberdade. Com isso, tem-se que o número ótimo de defasagens é $z=1$.

Após serem estabelecidas as condições necessárias para testar as hipóteses nulas de causalidade de preços entre os principais estados brasileiros produtores de leite, $e=1$ e $z=1$, com o que definiu-se o modelo VAR (2). Para tanto, melhor estabelecer as relações causais foram estimados modelos bivariados e também multivariados, conforme exposto na Tabela 5.

Tabela 5: Resultados dos testes de Causalidade de Toda e Yamamoto (1995) para as séries de preços do leite pago ao produtor dos principais estados produtores.

\begin{tabular}{c|cccc}
\hline Séries relacionadas & $\begin{array}{c}\text { Modelo } \\
\text { Bivariado } \\
\text { (F-statistic) }\end{array}$ & Hipótese nula & $\begin{array}{c}\text { Modelo } \\
\text { Multivariado } \\
\text { (F-statistic) }\end{array}$ & Hipótese nula \\
\hline Goiás>Bahia & $15.21781 * * *$ & $H_{0}: c_{4}=0$ & 0.006084 & $H_{0}: c_{4}=0$ \\
Minas Gerais>Bahia & $27.58019 * * *$ & $H_{0}: c_{4}=0$ & 1.518178 & $H_{0}: c_{6}=0$
\end{tabular}




\begin{tabular}{|c|c|c|c|c|}
\hline $\begin{array}{c}\text { Paraná>Bahia } \\
\text { Rio Grande do Sul>Bahia } \\
\text { São Paulo }>\text { Bahia }\end{array}$ & $\begin{array}{l}4.956596 * * \\
\mathbf{2 1 . 5 3 1 1 6} * * * \\
23.66368 * * *\end{array}$ & $\begin{array}{l}H_{0}: c_{4}=0 \\
H_{0}: c_{4}=0 \\
H_{0}: c_{4}=0\end{array}$ & $\begin{array}{c}0.129709 \\
\mathbf{5 . 8 5 6 9 2 6}^{* * * *} \\
0.893109\end{array}$ & $\begin{array}{c}H_{0}: c_{8}=0 \\
H_{0}: c_{10}=0 \\
H_{0}: c_{12}=0\end{array}$ \\
\hline Bahia>Goiás & 0.113242 & $H_{0}: c_{4}=0$ & $4.080014 * *$ & $H_{0}: c_{4}=0$ \\
\hline Minas Gerais>Goiás & $10.38866 * * *$ & $H_{0}: c_{4}=0$ & $4.171071 * *$ & $H_{0}: c_{6}=0$ \\
\hline Paraná>Goiás & $30.18769 * * *$ & $H_{0}: c_{4}=0$ & $15.34632 * * *$ & $H_{0}: c_{8}=0$ \\
\hline Rio Grande do Sul>Goiás & $10.64526^{* * *}$ & $H_{0}: c_{4}=0$ & 2.260579 & $H_{0}: c_{10}=0$ \\
\hline São Paulo>Goiás & $4.263653 * *$ & $H_{0}: c_{4}=0$ & 1.430615 & $H_{0}: c_{12}=0$ \\
\hline Goiás>Minas Gerais & $30.44634 * * *$ & $H_{0}: c_{4}=0$ & $16.36540 * * *$ & $H_{0}: c_{4}=0$ \\
\hline Bahia>Minas Gerais & $6.682584 * * *$ & $H_{0}: c_{4}=0$ & 0.824049 & $H_{0}: c_{6}=0$ \\
\hline Paraná>Minas Gerais & $10.86586 * * *$ & $H_{0}: c_{4}=0$ & $9.877895 * * *$ & $H_{0}: c_{8}=0$ \\
\hline $\begin{array}{c}\text { Rio Grande do Sul>Minas } \\
\text { Gerais }\end{array}$ & $22.05685 * * *$ & $H_{0}: c_{4}=0$ & $6.664559 * * *$ & $H_{0}: c_{10}=0$ \\
\hline São Paulo>Minas Gerais & $9.615535^{* * *}$ & $H_{0}: c_{4}=0$ & 0.406411 & $H_{0}: c_{12}=0$ \\
\hline Minas Gerais>Paraná & $9.077237 * * *$ & $H_{0}: c_{4}=0$ & $8.020242 * * *$ & $H_{0}: c_{4}=0$ \\
\hline Goiás>Paraná & $15.28465 * * *$ & $H_{0}: c_{4}=0$ & $5.864971 * * *$ & $H_{0}: c_{6}=0$ \\
\hline Bahia>Paraná & 0.025701 & $H_{0}: c_{4}=0$ & 0.563882 & $H_{0}: c_{8}=0$ \\
\hline Rio Grande do Sul>Paraná & $5.666529 * * *$ & $H_{0}: c_{4}=0$ & $7.255462 * * *$ & $H_{0}: c_{10}=0$ \\
\hline São Paulo>Paraná & $7.626258 * * *$ & $H_{0}: c_{4}=0$ & 0.246773 & $H_{0}: c_{12}=0$ \\
\hline Paraná>Rio Grande do Sul & $9.574682 * * *$ & $H_{0}: c_{4}=0$ & $6.547377^{* * * *}$ & $H_{0}: c_{4}=0$ \\
\hline Minas Gerais>Rio Grande do Sul & $17.07929 * * *$ & $H_{0}: c_{4}=0$ & 1.674698 & $H_{0}: c_{6}=0$ \\
\hline Goiás>Rio Grande do Sul & $18.82740 * * *$ & $H_{0}: c_{4}=0$ & $3.403913 * *$ & $H_{0}: c_{8}=0$ \\
\hline Bahia>Rio Grande do Sul & 0.025527 & $H_{0}: c_{4}=0$ & 0.736679 & $H_{0}: c_{10}=0$ \\
\hline São Paulo>Rio Grande do Sul & $12.49713 * * *$ & $H_{0}: c_{4}=0$ & 0.067464 & $H_{0}: c_{12}=0$ \\
\hline Rio Grande do Sul>São Paulo & $12.22115 * * *$ & $H_{0}: c_{4}=0$ & $4.438357 * *$ & $H_{0}: c_{4}=0$ \\
\hline Paraná >São Paulo & $5.778185 * * *$ & $H_{0}: c_{4}=0$ & $6.101624 * * *$ & $H_{0}: c_{6}=0$ \\
\hline Minas Gerais> São Paulo & $14.79020 * * *$ & $H_{0}: c_{4}=0$ & $5.188026^{* *}$ & $H_{0}: c_{8}=0$ \\
\hline Goiás>São Paulo & $12.90602 * * *$ & $H_{0}: c_{4}=0$ & $4.600460 * *$ & $H_{0}: c_{10}=0$ \\
\hline Bahia>São Paulo & 0.332666 & $H_{0}: c_{4}=0$ & 0.620763 & $H_{0}: c_{12}=0$ \\
\hline
\end{tabular}

Fonte: Elaborado pelos autores (2016).

Nota: A letra c estimada na hipótese nula indica o coeficiente restringido através do Teste de Wald.

Com o modelo bivariado identificou-se que o preço pago aos produtores no estado da Bahia, recebe influência dos preços de todos os outros estados incluídos na análise. Especificamente, verificou-se que a hipótese nula de não-causalidade de Toda e Yamamoto (1995) foi rejeitada a um nível de 1\% de significância para os estados de Goiás, Minas Gerais, Rio Grade do Sul e São Paulo, já para o estado de Paraná, com significância estatística de 5\%. Este resultado justifica-se pelo fato de que a Bahia, dentre os estados analisados, tem menor participação na produção total de leite in natura, cerca de 3,4\%, além de ser um importador líquido de leite e derivados (ALICEWEB, 2016). 
Porém, quando se trata do modelo multivariado, onde as séries de preços de todos os estados foram incluídas no modelo, a hipótese nula de não-causalidade de Toda e Yamamoto (1995) foi rejeitada apenas para a série de preços do estado do Rio Grande do Sul, confirmando o resultado encontrado no modelo bivariado. Estas estimativas sugerem que as flutuações ocorridas nos preços pagos ao produtor de leite no estado sulista influenciam (precedem) as variações observadas nos preços praticados pelo estado da Bahia. Esse resultado justifica-se pelo nível de produção dos dois estados, em que o estado gaúcho é mais representativo, quando comparado ao estado baiano.

Ressaltando que, embora os produtores de leite do estado da Bahia se preocupem em aumentar a produtividade de seus rebanhos para conseguir maior representatividade no cenário nacional, ainda enfrentam dificuldades ligadas à genética, alimentação e falta de tecnologias empregadas na atividade. Essas deficiências aliadas ao baixo conhecimento em gestão por parte dos produtores rurais se refletem em uma baixa produtividade e, assim pouca representatividade no mercado interno de leite in natura (PATÊS, 2011).

$\mathrm{Na}$ análise dos preços pagos ao produtor no estado de Goiás, verificam-se resultados semelhantes aos encontrados para o estado da Bahia, dado que a hipótese nula de não-causalidade de Toda e Yamamoto (1995) foi rejeitada para as séries de todos os demais estados, com exceção da série do estado da Bahia, quando estimado um modelo bivariado. Neste caso, indica-se a existência de uma relação unidirecional de causalidade, em que as variações nos preços de Goiás causam as variações nos preços da Bahia, porém o contrário não é observado, justificando-se pela diferença na quantidade de leite produzida das duas regiões.

As relações de precedência de preços encontradas quando estimados os modelos aos pares foi confirmado com o modelo multivariado nos casos de Minas Gerais e Paraná. Além disso, as oscilações nos preços do leite in natura pago ao produtor do estado de Goiás recebem influências das variações ocorridas em outros dois estados.

Quando analisado os preços no estado de Minas Gerais, identifica-se uma causalidade bidirecional com os preços praticados no estado de Goiás. Esse resultado justifica-se pela proximidade dos dois estados e também pela representatividade de produção que os mesmos possuem na produção total de leite. Outro ponto a ser destacado, é que os dois estados, juntamente com os valores praticados no estado de São 
Paulo, possuem preços pagos ao produtor mais elevados com relação a outros estados brasileiros (ver Tabela 2). Ainda, para o estado de Minas Gerais, a um nível de $1 \%$ de significância, os preços do estado do Paraná indicam preceder os preços de leite no estado mineiro.

O estado do Paraná apresenta comportamento diferente dos outros estados, visto que os preços pagos aos produtores de leite paranaenses recebem influência de todos os outros estados, com exceção do estado de São Paulo, corroborando os resultados encontrados por Dias et al., (2008). Essa influência foi confirmada tanto pelo modelo bivariado quanto pelo modelo multivariado. Evidência que se justifica devido à necessidade que o estado possui em importar, tanto leite in natura, quanto derivados, para suprir a demanda interna (ALICEWEB, 2016). Nesse caso, recebe influência dos preços dos estados exportadores.

Ainda na região sul, os preços de leite in natura praticado no estado do Rio Grande do Sul, por meio do teste de Causalidade de Toda e Yamamoto (1995), demonstraram receber influência dos preços dos estados do Paraná e Goiás, da mesma forma que os resultados encontrados por Dias et al., (2008). Esses resultados foram confirmados nos dois modelos estimados. Ressaltando que há uma causalidade bidirecional com relação ao estado do Paraná, fato que justifica-se pela proximidade dos dois estados, e também pelas características de produção muito parecidas presentes nos estados da região sul (TRICHES, 2011).

Da mesma forma que o estado do Paraná, o estado de São Paulo também recebe influência nos preços de leite pago ao produtor dos preços nos outros estados analisados, com exceção da Bahia, confirmado pelos dois modelos - bivariado e multivariado. Esse resultado pode ser explicado pela localização do estado de São Paulo, e também por contar com o Porto de Santos, que é outro porto responsável pela entrada e saída de produtos agrícolas e pecuários (CONAHB, 2015). Embora não possua relevância na produção de leite in natura, o estado de São Paulo é responsável por grande parte das exportações de leite in natura e derivados. Assim, necessita de matéria-prima de outros estados para processar e exportar derivados lácteos.

Sintetizando, na Figura 2 estão expostos os principais resultados encontrados na estimação da causalidade de Toda e Yamamoto (1995) para os preços dos estados analisados. 
Figura 2: Principais resultados de causalidade encontrados.

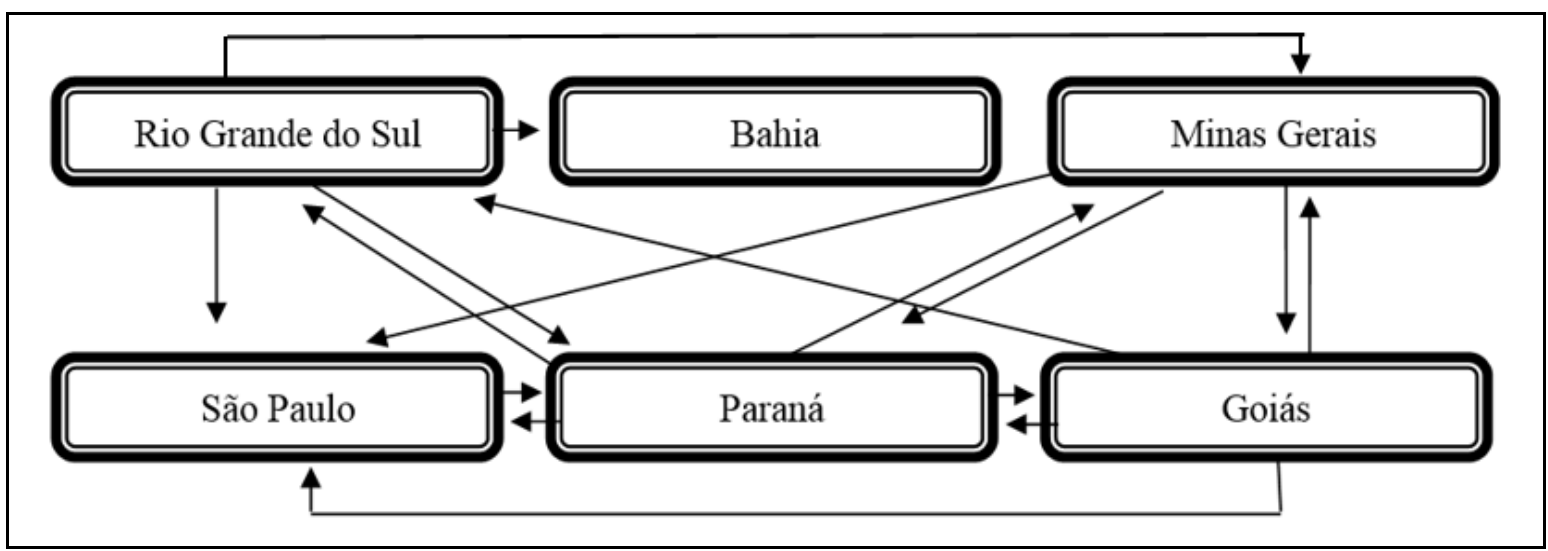

Fonte: Elaborado pelos autores (2016).

Em resumo, os resultados indicaram a existência de causalidade de preços bidirecional entre os preços de leite in natura pagos aos produtores dos estados de Minas gerais e Goiás, Paraná e Rio Grande do Sul, Minas Gerais e Paraná e São Paulo e Paraná.

Nesse caso, os estados que são considerados formadores de preços do leite in natura são Paraná, Minas Gerais e Rio Grande do Sul. Isso, pois exercem influência nos preços dos demais estados. Da mesma forma que o estado de São Paulo figura como tomador de preços, já que recebe influência de praticamente todos os outros estados em análise.

\section{CONCLUSÕES}

Ao longo dos anos, o setor brasileiro de lácteos passou por diversas mudanças, mas a principal delas foi o fim do tabelamento de preços ocorrido na década de 1990. A partir desse período, a produção de leite passou a ser desenvolvida de diferentes formas pelo Brasil, induzindo a formação de um mercado bastante heterogêneo.

Atualmente, existem diferentes estruturas produtivas nas regiões, bem como níveis de produtividade e preços distintos. Isso se deve pelo fato de que em algumas regiões o nível de tecnologia empregado na produção de leite é mais elevado quando comparado a outras. Além disso, conhecimentos em gestão, qualidade e incentivos 
governamentais à produção de leite contribuem para as diferenças produtivas nas cinco regiões brasileiras.

Neste cenário, os estados de Minas Gerais, Paraná, Rio Grande do Sul, Goiás, Bahia e São Paulo são aqueles que apresentam maior representatividade na produção de leite no Brasil. Esta configuração produtiva sugere que pode haver a influência de algumas regiões na formação dos preços de outras, aspecto que fundamentou o objetivo de analisar a relação de preços do leite in natura entre os principais estados brasileiros produtores.

Os resultados possibilitaram verificar uma relação consistente de causalidade bidirecional entre os estados de Goiás e Paraná, Goiás e Minas Gerais, Minas Gerais e Paraná, Rio Grande do Sul e Paraná e São Paulo e Paraná. Ademais, a partir de ambos os modelos bivariado e multivariado, constatou-se a causalidade unidirecional do Rio Grande do Sul para a Bahia, Minas Gerais e São Paulo, e de Goiás para o Rio Grande do Sul e para São Paulo, e ainda, de Minas Gerais para São Paulo. Logo, tem-se que os estados do Paraná e de São Paulo recebem influência dos demais estados produtores.

Com os resultados, indica-se que os estados de Paraná, Minas Gerais e Rio Grande do Sul possuem características de formadores de preços, já que exercem influência sobre os preços dos demais estados em análise. Já os preços praticados pelo estado de São Paulo são impactados pelos preços dos outros estados produtores de leite, assim, figura como tomador de preços.

Como limitações do trabalho indicam-se a falta de dados de outros estados brasileiros, de forma a possibilitar uma comparação mais detalhada entre regiões, visto que as regiões norte e nordeste não foram incluídas. Para estudos futuros, entre outras questões, sugere-se análise sobre os preços dos produtos lácteos com relação aos preços pagos ao produtor pelo leite in natura.

\section{REFERÊNCIAS}

ALICEWEB, ANÁLISE DAS INFORMAÇÕES DE COMÉRCIO EXTERIOR. Consultas. Disponível em <http://aliceweb.desenvolvimento.gov.br/> . Acesso em jul 2016.

ALIMI, S. R; OFONYELU, C. C. TODA-YAMAMOTO Causality Test between money market interest rate and expected inflation: the fisher hypothesis revisited. 
European Scientific Journal. March 2013 edition vol.9, No.7 ISSN: 1857 - 7881 (Print) e -ISSN 1857- 7431.

ARRAES, R. A.; SOUZA FILHO, E. Externalidades e formação de preços no mercado imobiliário urbano brasileiro: um estudo de caso. Economia aplicada, São Paulo, v. 12, n. 2, p. 289-319, abril-junho 2008.

BANKÚTI, I. B. Sistema agroindustrial do leite: alterações de mercado e propostas de adequação ao produtor. Disponível em:

$<$ http://www.iepec.com/noticia/sistemaagroindustrial-do-leite-alteracoes-de-mercado-epropostas-de-adequacao-ao-produtor>. Acesso em: 02 abr. 2016.

BARROS, F. L. A.; DE LIMA, J. R. F.; FERNANDES, R. A. S. ANÁLISE DA

ESTRUTURA DE MERCADO NA CADEIA PRODUTIVA DO LEITE NO

PERÍODO DE 1998 A 2008. Revista de Economia e Agronegócio-REA, v. 8, n. 2,

2010.

BARROS, G. S. A. D. C., SBRISSIA, G. F., SPOLADOR, H. F. S., \& PONCHIO, L. A. (2004). Mudanças estruturais na cadeia do leite Reflexos sobre os preços. Revista de Política Agrícola, 13(3), 13-26.

BORGES, M. S.; CASTRO, M. C. D.; GUEDES, C. A. M.; ALIMONDA, H. A. Modernização, Trabalho e Produtividade na Pequena Produção Leiteira na Argentina e no Brasil. Revista ADM.MADE, Rio de Janeiro, ano 14, v.18, n.1, p.12-31, janeiro/abril, 2014.

BORTOLETO, E. WILKINSON, J. Competitividade, inovação e demandas tecnológicas no sistema agroindustrial do Mercosul ampliado - lácteos. Anais... CONGRESSO BRASILEIRO DE ECONOMIA E SOCIOLOGIA RURAL, 38., 2000, Rio de Janeiro -RJ. Anais. SOBER, 2000.

CAMPOS, K. C.; PIACENTI, C. A. Agronegócio do leite: Cenário atual e perspectivas. Anais... XLV CONGRESSO DA SOBER - Sociedade de Economia, Administração e Sociologia Rural. 22 a 25 de julho de 2007. Londrina-PR.

CENTRO DE ESTUDOS AVANÇADOS EM ECONOMIA APLICADA - CEPEA. Boletim do leite. Ano $22 \mathrm{n}^{\circ} 249$ - Fevereiro de 2016. Disponível em:

<http://cepea.esalq.usp.br/leite/boletim/249.pdf>. Acesso em: 02 abr. 2016.

DIAS, D. F., KRETZMANN, C. K., ALVES, A. F., PARRÉ, J. L. (2007). Análise da transmissão de preço para o leite paranaense utilizando modelos de séries temporais.

Revista em Agronegócio e Meio Ambiente, 1(1), 09-24.

DICKEY, D.A.; FULLER, W.A. Likelihoodratiostatistics for autoregressive time series with a unit root. Econometrica, 49:1057-1072, 1981.

EMIRMAHMUTOGLU, F.; KOSE, N. Testing for Granger causality in heterogeneous mixed panels. Department of Econometrics, Gazi University, IncitasiSokag1 No:4, 06500 Besevler, Ankara, Turkey. EconomicModelling 28 870-876, (2011). 
EMPRESA BRASILEIRA DE PESQUISA AGROPECUÁRIA - EMBRAPA. Embrapa Gado de Leite. Sistemas de Produção. Disponível em:

<http://www.cnpgl.embrapa.br/sistemaproducao/>. Acessoem: 02 abr. 2016.

ENGLE, R. F.; GRANGER; C W J; Co-integration and error correction: representation, estimation, and testing. Econometrica: journaloftheEconometric. v. 55, n. 2, p. 251276, 1987.

FERNANDES, R. A. S., BRAGA, M. J., LIMA, J. E. D. (2008). Elasticidade Na Transmissão E Formação Espacial De Preços De Leite Ao Produtor Nos Principais Estados Brasileiros. In 46th Congress, July 20-23, 2008, Rio Branco, Acre, Brasil (No. 108577). Sociedade Brasileira de Economia, Administração e Sociologia Rural (SOBER).

FOCHEZATTO, A.; KOSHIYAMA, D.; ALENCASTRO, D.; Testando Relações de Causalidade entre Comércio Externo e Crescimento Econômico em Países da América Latina: Evidências de Dados em Painel e Séries Temporais. Revista EconomiA, Brasília(DF), v.11, n.3, p.597-629, set/dez 2010.

FOCHEZATTO, A.; KOSHIYAMA, D.; ALENCASTRO, D. O aumento da lucratividade expande a acumulação de capital? uma análise de causalidade de Granger para países da OCDE. Revista economia contemporânea. vol.13 no.3 Rio de Janeiro Sept./Dec. 2009.

GRANGER, C. W. J. Investigating Causal Relations by Econometric Models and Cross-spectral Methods. Econometrica, Vol. 37, No. 3.(1969).

GREENE, Q. W. H. Econometric analysis. Fifht edition. New York University. July 10, (2002).

INSTITUTO BRASILEIRO DE GEOGRAFIA E ESTATÍSTICA - IBGE. Pesquisa Pecuária Municipal - 2014. Disponível em:

<http://ibge.gov.br/home/estatistica/indicadores/agropecuaria/producaoagropecuaria/aba te-leite-couro-ovos_201402_publ_completa.pdf>. Acesso em: 02 abr. 2016.

MURAKAMI, P. N. Causalidade de Granger em medidas de risco. 2011. Tese de Doutorado. Universidade de São Paulo. Disponível em:

<http://www.teses.usp.br/teses/disponiveis/45/45133/tde-14072011-221932/en.php>. Acesso em: 30 mar. 2016.

PATÊS, N. M da S. Diagnóstico participativo da pecuária leiteira no sudeste da Bahia. (Tese). Programa de Pós-graduação em Zootecnia. Universidade Estadual do Sudoeste da Bahia. Iapetinga, BA: 2011.

RIBEIRO, S. G. B.; de OLIVEIRA, S. C.; SCALCO, A. R.; de Barros PINTO, L. O COMPORTAMENTO DO PREÇO DO LEITE TIPO C PAGO AO PRODUTOR NO ESTADO DE SÃO PAULO CONFRONTADO COM A REALIDADE DOS 


\section{PRODUTORES DA MICRORREGIÃO DE MARÍLIA. Revista Brasileira de}

Engenharia de Biossistemas, v. 5, n. 2, p. 117-127, 2011.

SOCIEDADE NACIONAL DA AGRICULTURA. Notícias. Consumo de lácteos perde fôlego no País. 2015. Disponível em:<http://sna.agr.br/consumo-de-lacteos-perdefolego-no-pais/>. Acesso em: 07 mar. 2016.

STOCK, J. H. WATSON, M. W. Econometria. Pearson Education do Brasil. 2004.

SULKU, S. N. Causality Relation between Real Exchange Series and Emerging Stock Markets: Panel Common Factor Analysis. Journal of Applied Economics and Business Research. JAEBR, 1(3): 130-148 (2011).

TODA, H.Y.; YAMAMOTO, T. Statistical inference in Vector Autoregressions with possibly integrated processes. JournalofEconometrics, 66, 225-250. (1995).

TRICHES, E. Importância da atividade leiteira na agricultura familiar e uma análise na propriedade Ghion-Marau-RS. 2011.Universidade Federal do Rio Grande do Sul. Curso de Tecnólogo em Planejamento e Gestão para o Desenvolvimento Rural a Distância. Disponível em: <http://hdl.handle.net/10183/54730>. Acesso em: 30 mar. 2016.

YAMAGUCHI, L. C. T; ARAÚJO, L. F. O. Preços de produto e insumo no mercado de leite: um teste de causalidade. Revista Eletrônica de Economia, n. 6, 2005.

WOOLDRIDGE, J. M. IntroductoryEconometrics-AModernAproach. 2ed., Ohio, Thomson South-Western, 2003. 